\title{
EIT: EXTREME-ULTRAVIOLET IMAGING TELESCOPE FOR THE SOHO MISSION
}

\author{
J.-P. DELABOUdiniÈRE, G. E. ARTZNER, J. BRUNAUD, A. H. GABRIEL, \\ J. F. HOCHEDEZ, F. MILLIER and X. Y. SONG \\ Inst. d'Astrophysique Spatiale, Université Paris XI, 91405 Orsay Cedex, France \\ B. AU, K. P. DERE, R. A. HOWARD, R. KREPLIN, D. J. MICHELS and \\ J. D. MOSES \\ Naval Research Laboratory, Washington, DC 20375, USA
}

J. M. DEFISE, C. JAMAR and P. ROCHUS

Centre Spatial de Liège, Liège, Belgium

J. P. CHAUVINEAU and J. P. MARIOGE

Institut d'Optique Théorique et Appliquée, 91403 Orsay, France

R. C. CATURA, J. R. LEMEN, L. SHING and R. A. STERN

Lockheed Palo Alto Research Laboratory, Palo Alto, CA 94304, USA

J. B. GURMAN and W. M. NEUPERT*

NASA/Goddard Space Flight Center, Greenbelt, MD, USA

A. MAUCHERAT

Laboratoire d'Astronomie Spatiale, Marseille, France

and

F. CLETTE, P. CUGNON and E. L. VAN DESSEL

Observatoire Royal de Belgique, Brussels, Belgium

\begin{abstract}
The Extreme-ultraviolet Imaging Telescope (EIT) will provide wide-field images of the corona and transition region on the solar disc and up to $1.5 \mathrm{R}_{\odot}$ above the solar limb. Its normal incidence multilayer-coated optics will select spectral emission lines from Fe IX $(171 \AA)$, Fe XII $(195 \AA), F e$ XV $(284 \AA)$, and He II (304 $\AA)$ to provide sensitive temperature diagnostics in the range from $6 \times 10^{4} \mathrm{~K}$ to $3 \times 10^{6} \mathrm{~K}$. The telescope has a $45 \times 45$ arcmin field of view and 2.6 arcsec pixels which will provide approximately 5 -arcsec spatial resolution. The EIT will probe the coronal plasma on a global scale, as well as the underlying cooler and turbulent atmosphere, providing the basis for comparative analyses with observations from both the ground and other SOHO instruments. This paper presents details of the EIT instrumentation, its performance and operating modes.
\end{abstract}

Key words: Solar X-rays-Solar EUV-Multilayer telescope

\section{Introduction}

The EIT (Delaboudinière et al. 1989a, 1989b, Clette et al. 1995) is designed to provide full-disk images of the solar transition region and the inner solar corona to $1.5 \mathrm{R}_{\odot}$. It will do this by employing the relatively new technology of multilayer normal incidence extreme-ultraviolet (EUV) optics (Spiller,

* Present address is SEL/NOAA 325 Broadway, Boulder, CO 80303

Solar Physics 162: 291-312, 1995.

(C) 1995 Kluwer Academic Publishers. Printed in Belgium.

C Kluwer Academic Publishers • Provided by the NASA Astrophysics Data System 
1994). By dividing the telescope mirrors into quadrants it is possible to isolate the emissions from narrow temperature ranges by 'tuning' the coatings of each quadrant to the desired wavelength band (Table I).

TABLE I

EIT Bandpasses.

\begin{tabular}{cccc}
\hline Wavelength & Ion & Peak Temperature & Observational Objective \\
\hline $304 \AA$ & He II & $8.0 \times 10^{4} \mathrm{~K}$ & chromospheric network; coronal holes \\
$171 \AA$ & Fe IX-X & $1.3 \times 10^{6} \mathrm{~K}$ & $\begin{array}{c}\text { corona/transition region boundary; } \\
\text { structures inside coronal holes }\end{array}$ \\
$195 \AA$ & Fe XII & $1.6 \times 10^{6} \mathrm{~K}$ & quiet corona outside coronal holes \\
$284 \AA$ & Fe XV & $2.0 \times 10^{6} \mathrm{~K}$ & active regions \\
\hline
\end{tabular}

The primary scientific objective of the EIT is to study the dynamics and evolution of coronal structures over a wide range of time scales, sizes and temperatures to bring new insights into the study of mechanisms responsible for coronal heating and solar wind acceleration. Images in coronal lines will show coronal loops and their magnetic topology and possibly its changes caused by magnetic reconnection. The He II transition region images will indicate the magnetic footpoints of the coronal loops and outline the bases of coronal holes which are the locations of open magnetic fields where high speed solar wind streams originate. Such images will provide the connection between structures observed on the solar disk and those seen above the limb by LASCO and UVCS. Time series of images in limited areas will also define the global dynamics of structures that are simultaneously observed with the spectroscopic instruments (CDS and SUMER) which have limited fields of view.

EIT's images in He II provide sensitivity to solar structures an order of magnitude lower in temperature than the spectacular imagery obtained by the Soft X-ray Telescope (SXT) (Tsuneta et al. 1991 and see special issue on initial results from Yohkoh in Publ. Astron. Soc. Japan, 1992, 44, No. 5) on the Yohkoh mission, while maintaining overlap with that instrument's capabilities in the 0.6 to $3 \mathrm{MK}$ range. If present plans are realized, the EIT and SXT operations will overlap by as much as 18 months, allowing comparative analyses of their contemporaneous images with temperature coverage from 0.06 to $100 \mathrm{MK}$.

EIT observations from SOHO's position at the $L 1$ Lagrangian point will allow data continuity uninterrupted by earth occultations that occur in observations from low earth orbit. This will allow access to a frequency domain in dynamical analyses that has been previously inaccessible in soft $\mathrm{X}$-ray and EUV observations due to periodic gaps in the data. 


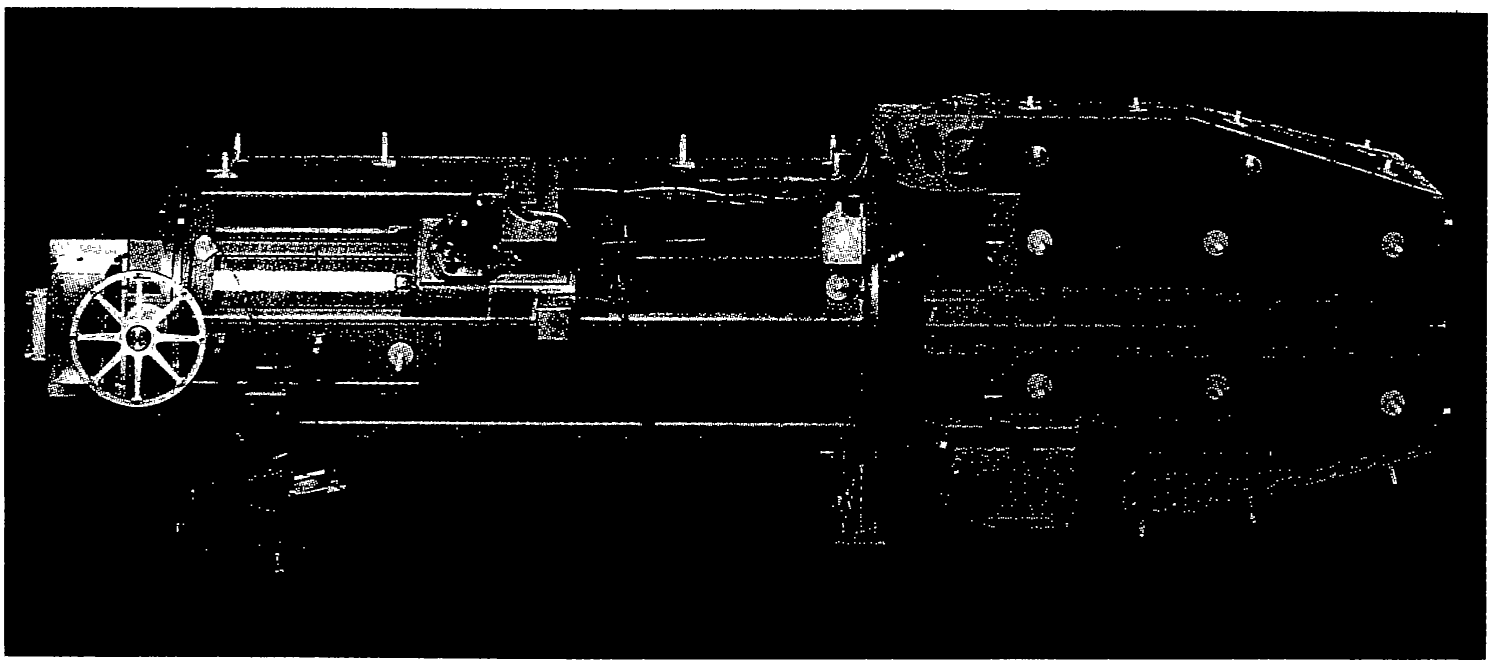

Fig. 1. Photograph showing the EIT flight instrument.

\section{Instrumentation}

\subsection{Instrument Overview}

The EIT is a telescope of Ritchey-Chretien design that will obtain full-disk images in four narrow passbands with a field of view 45 arcmin square and a spatial resolution limited only by the 2.6 arcsec pixel size of the CCD image sensor. The EIT uses four separate multilayers that are deposited on matched quadrants of both the primary and secondary mirrors of the telescope (Table I). The bandpasses are defined through interference effects arising in the multilayer coatings. A rotating mask allows only a single multilayer-coated quadrant of the telescope to be illuminated by the Sun at any time. All of the multilayers are fabricated from alternating layers of

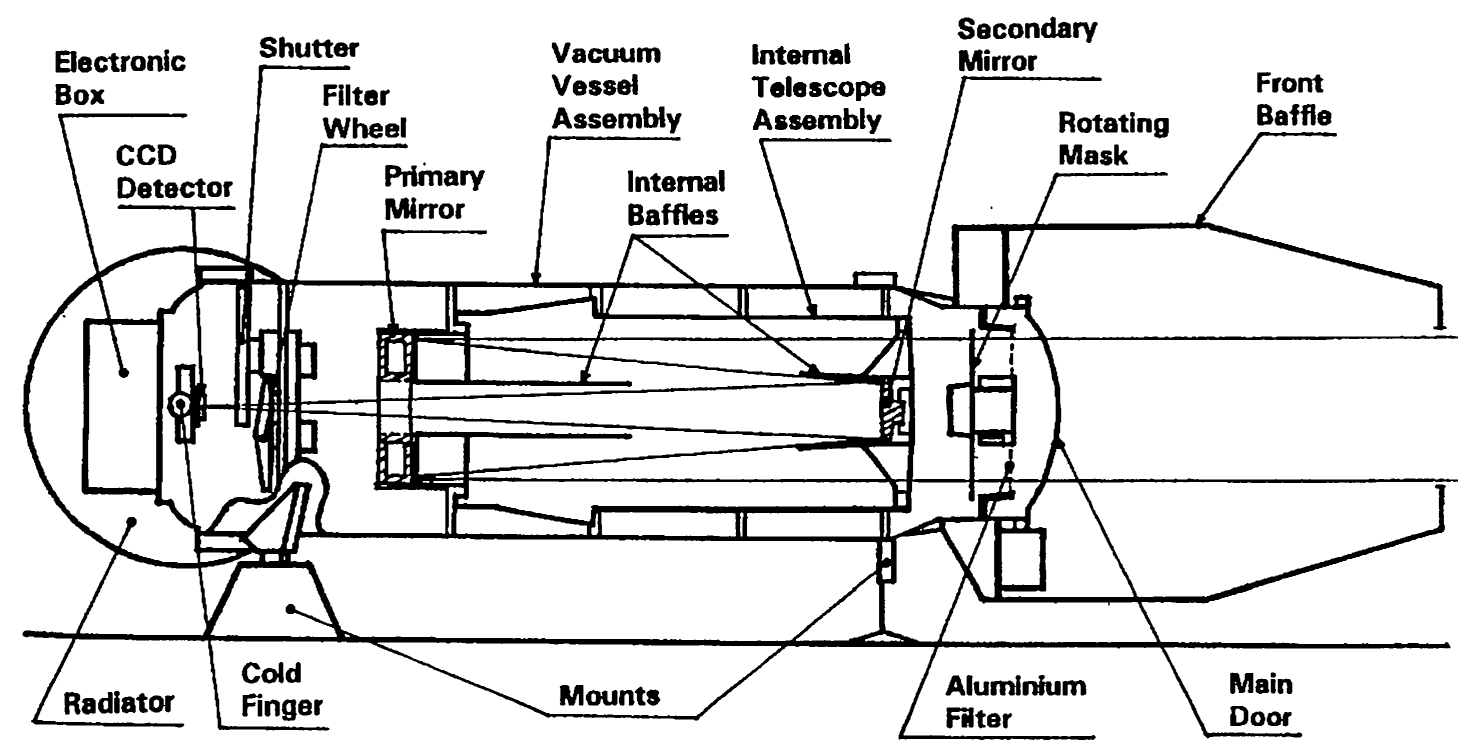

Fig. 2. Schematic of the EIT indicating its major subsystems. 
molybdenum and silicon. A photograph of the flight instrument appears in Figure 1 and a schematic of EIT is shown in Figure 2, indicating its major subsystems. The telescope is contained in a vacuum enclosure for cleanliness and to reduce acoustical loads on the thin-film filters during launch. The main door at the front of the telescope is opened after spacecraft outgassing and may be re-closed when thrusters are fired for SOHO station keeping. A heat rejection filter composed of $700 \AA$ of cellulose sandwiched between $1500 \AA$ thick films of $\mathrm{Al}$ covers the EIT entrance aperture.

The image from a selectable single EIT quadrant is focused on a backilluminated CCD whose detection efficiency at extreme-ultraviolet (EUV) wavelengths has been enhanced by a special fabrication process (Catura and Shing, 1993). The CCD is cooled to about $-80 \mathrm{C}$ by a cold finger attached to a passive radiator facing deep space. Since each quadrant of the optics forms an image on a single CCD the registration of the images from the four passbands is accomplished automatically. The telescope filter wheel near the focal plane has two filters to block long wavelength solar emission, an open position and two opaque $\mathrm{Al}$ screens that block the upper and lower one third of the reflected solar image, respectively, from reaching the CCD. These opaque screens allow the CCD to be operated in a frame transfer mode to provide a redundancy to shutter operation, but image only about one quarter of the Sun at each exposure. One exposed quarter of the CCD is transferred to the occulted section in a time of $50 \mathrm{~ms}$. The CCD camera electronics are behind and adjacent to the CCD, but are hermetically sealed from it for purposes of cleanliness.

The computer and electronics that control the EIT instrument are contained in the LASCO electronic box (LEB), with a single interface to the spacecraft command and telemetry system. Consequently, the LASCO and EIT instruments share a combined telemetry rate of $1+4.2 \mathrm{Kbps}=5.2 \mathrm{Kbps}$. Operationally, the 3 LASCO telescopes and the EIT can be viewed as a single instrument package of 4 telescopes. This provides considerable flexibility in operating the EIT/LASCO combination, but also introduces a few constraints. A telemetry rate of $26.2 \mathrm{kbps}$ can be made available for short time periods from the CDS and SUMER instruments for purposes of obtaining timely images for planning their observing programs. Further details of EIT are given in Table II.

\subsection{Optics}

The telescope is a modification of the wide-field Ritchey-Chretien, introduced by Bottema and Woodruff (1971), which can be used with a sizeable tilt of the secondary mirror to facilitate alignment or even permit image tracking. The exact parameters of the EIT optics have been discussed by Delaboudinière et al. (1989b). The shape of the primary and secondary mir- 
TABLE II

EIT Characteristics.

\begin{tabular}{|c|c|}
\hline \multicolumn{2}{|c|}{ Telescope (Ritchey-Chretien) } \\
\hline Mirrors & Multilayer-coated Zerodur \\
\hline Multilayers & Mo-Si tuned to different $\lambda \mathrm{s}$ in each quadrant \\
\hline Bandpass centers & $171 \AA, 195 \AA, 284 \AA$ and $304 \AA$ \\
\hline Bandpass selection & Rotatable open quadrant shutter \\
\hline Effective focal length & $165.2 \pm 0.2 \mathrm{~cm}$ \\
\hline Primary diameter & $12 \mathrm{~cm}$ \\
\hline Geometrical area per quadrant & $13 \mathrm{~cm}^{2}$ \\
\hline \multicolumn{2}{|c|}{ CCD detector (cooled to about-80 C) } \\
\hline Architecture & $\begin{array}{l}\text { Three phase, multiphase-pinned, } \\
\text { back-illuminated, enhanced EUV sensitivity }\end{array}$ \\
\hline Array size & $1024 \times 1024(45 \times 45$ arcmin $)$ \\
\hline Pixel size & $21 \mu \mathrm{m}$ square $(2.6$ arcsec $)$ \\
\hline Full well & $>150,000$ electrons \\
\hline Readout rate & $5 \times 10^{4}$ pixels $/ \mathrm{sec}$ \\
\hline On-chip summation & e.g. $2 \times 2$ and $4 \times 4$ pixels \\
\hline Full frame readout time & $21 \mathrm{sec}$ \\
\hline \multicolumn{2}{|r|}{ Filters } \\
\hline Entrance Filter & $1500 \AA \mathrm{Al} / 700 \AA$ cellulose $/ 1500 \AA \mathrm{Al}$ \\
\hline Filter wheel: Pos. 0 & $1500 \AA \mathrm{Al}$ \\
\hline Pos. 1 & $1500 \AA \mathrm{Al} / 700 \AA$ cellulose $/ 1500 \AA \mathrm{Al}$ \\
\hline Pos. 2 & CCD bottom third blocked, top $1500 \AA \mathrm{Al}$ \\
\hline Pos. 3 & Open, no filter \\
\hline Pos. 4 & CCD top third blocked, bottom $1500 \AA \mathrm{Al}$ \\
\hline CCD stray light filter & $1500 \AA \mathrm{Al}$ adjacent to $\mathrm{CCD}$ \\
\hline \multicolumn{2}{|c|}{ Telemetry } \\
\hline Normal average rate & $1 \mathrm{Kbps}$ \\
\hline EIT/LASCO combined rate & $5.2 \mathrm{Kbps}$ \\
\hline Enhanced (from CDS and SUMER) & $26.2 \mathrm{Kbps}(30$ mins per $24 \mathrm{hrs})$ \\
\hline \multicolumn{2}{|l|}{ Data Compression } \\
\hline square root & 14 bits to 7 bits \\
\hline ADCT and Rice compressions & Up to a factor of 10 compression \\
\hline \multicolumn{2}{|c|}{ Full-frame Unsummed Image Transmission Times (at 7 bits/pixel compression): } \\
\hline Normal TM (5.2 Kbps) & $23.5 \mathrm{~min}-\max$ cadence of 1 every $2 \mathrm{hrs}$. \\
\hline Enhanced TM (short periods) & 4.7 mins - max cadence of 1 every 24 mins. \\
\hline \multicolumn{2}{|c|}{ Full-frame Summed $4 \times 4$ Pixels Transmission Times (at 7 bits/pixel compression): } \\
\hline Normal TM & 1.5 min - max cadence of 1 every 7.6 mins. \\
\hline Enhanced TM & $17.5 \mathrm{sec}-$ max cadence of 1 every 1.5 mins. \\
\hline
\end{tabular}




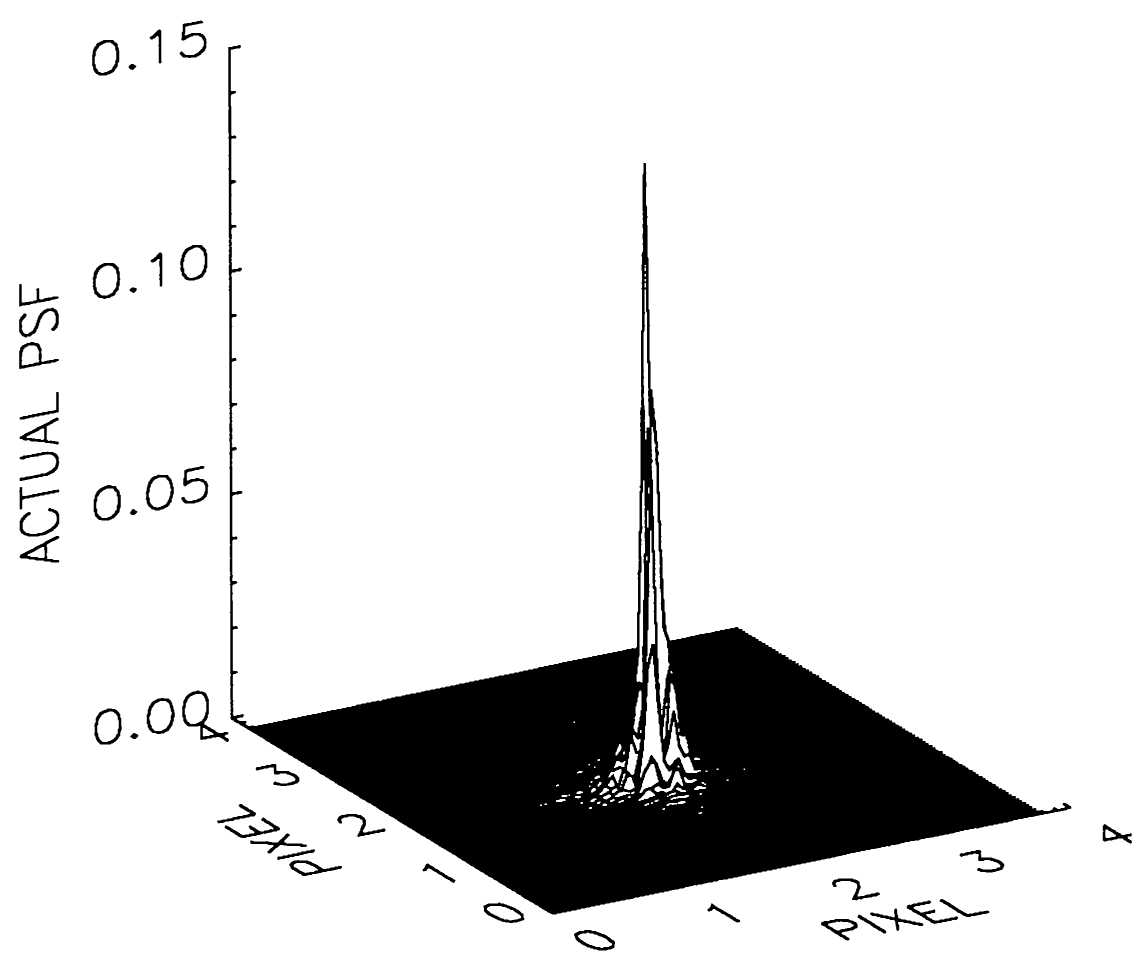

Fig. 3. EIT point spread function at $304 \AA$ inferred from wave front error measurements.

rors were accurately defined $(\lambda / 50)$ by a two step manufacturing process described by Chauvineau et al. (1991).

The telescope alignment was achieved and controlled by auto-collimation on a pinhole at the entrance of a ZYGO interferometer. The wave front error was mapped and measured by the same technique for each quadrant of the telescope. The different thicknesses of the multilayer stacks introduce phase shifts which are of no consequence for the images, since only one quadrant is used at a time.

From the measured wavefronts one can compute point spread functions (PSFs) at the operating wavelengths. An example for a wavelength of $304 \AA$ is shown in Figure 3. The PSFs are narrower than the pixel size of the CCD and by convolution with the square pixel shape one can deduce the angular resolution of the instrument on the solar disk. As shown in Figure 4, this convolution is very close to the nominal $2.6 \times 2.6$ arcsec angular size of the pixels. One pixel will indeed correspond to a very well defined area on the Sun. The telescope was also tested with a collimator in visible light and at the $2537 \AA$ emission line of mercury. Diffraction limits the "two-pixel" 


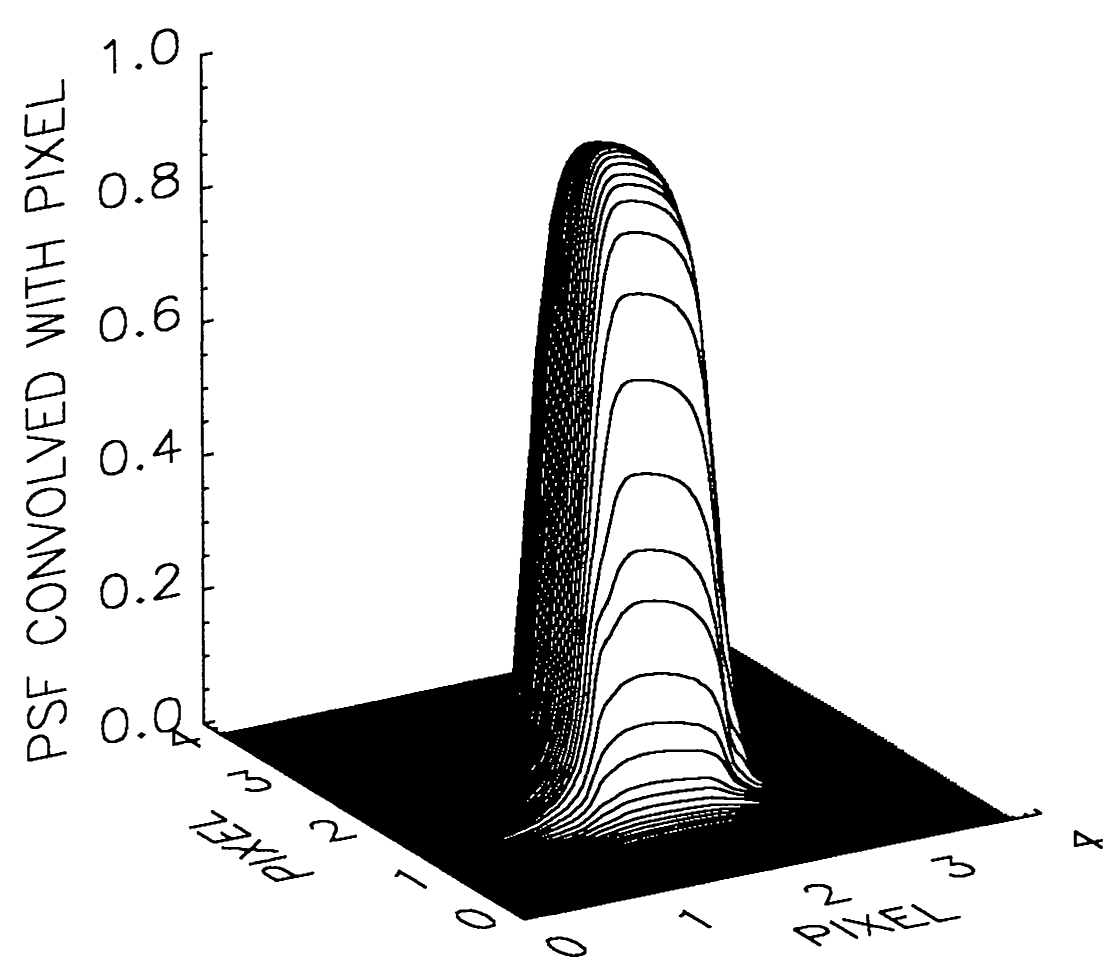

Fig. 4. Point spread function of Fig. 3 convolved with the $21 \mu \mathrm{m}$ square-sized pixel.

mirror performance to 4 arcsec in the first case and to $2 \operatorname{arcsec}$ as expected at the UV wavelength.

The thermal design of the optics was driven by two issues: control of the optical bench and maintaining the CCD in focus while cooling it to about $-80 \mathrm{C}$. Great attention was taken to control the spacing between the telescope mirrors to a tolerance of $7 \mu \mathrm{m}$ to maintain image quality. These two mirrors are mounted on each end of an aluminum cylinder, attached to the main structure by a unique central flange to avoid thermal stresses.

The on-ground optical alignment is optimized for a $20 \mathrm{C}$ environmental temperature. Once on orbit, due to possible inaccuracies in the thermal design and changing thermal properties with age, the equilibrium internal temperature may not be at $20 \mathrm{C}$ for all the observations. To address this problem, the thermal design has been calculated and tested so that the onorbit temperature of the internal structure is less than $20 \mathrm{C}$. The optical cylinder structure is wrapped with thermofoil heaters and includes a set of thermistors. Thus, the spacing between primary and secondary mirrors is maintained constant by heating the structure using active thermal control. 


\subsection{CCD Image Sensor}

The CCD used to record the images is a thinned, back-illuminated device fabricated by Scientific Imaging Technologies Inc. (SITe, formerly a group within Tektronix). The back surface has been processed to provide a high and stable EUV quantum efficiency (Moses, et al. 1993). The CCD is a $1024 \times 1024$ array of $21 \mu \mathrm{m}$ square pixels with gate implants to provide MultiPinned Phase (MPP) operation. This provides lower thermally generated dark current and improves the radiation hardness of the CCD. There are four readout ports, at the corners of the image array, with only one used at any time. The full-well capacity of a pixel is about 150,000 electrons and the charge transfer efficiency of the device is about 0.99995. The CCD is mounted in a special ceramic package with provision to position its surface accurately and reproducibly in the EIT focal plane. The CCD is cooled by passively radiating to deep space and the temperature is regulated at about $-80 \mathrm{C}$ by a small heater. This heater may be used with an additional higher wattage heater to warm the CCD above ambient temperature in order to evaporate any condensates that may have formed on its surface and that could degrade its EUV quantum efficiency. A temperature sensor is located on the ceramic package and another is present on the CCD itself.

\subsection{Camera and Control Electronics}

As indicated earlier, EIT is operated in concert with the LASCO instrument on SOHO. LASCO has three CCD cameras whose operations are identical to that of EIT, except that EIT requires a factor of 8 longer parallel transfer time due to the backside illumination. This adds only $0.43 \mathrm{sec}$ to the readout time, since most is spent in the serial transfers. Thus, EIT appears to the LASCO electronics box as a fourth LASCO camera and receives commands, data processing and telemetry access through that box and is allocated $20 \%$ of the available resources. The output of the camera is a 14 bit digital number (DN). The system gain is such that the least significant bit of each DN corresponds to 18 electrons per pixel detected in the CCD. A description of the cameras, control electronics, software and data processing is presented in the LASCO paper by Brueckner et al. (1995, in this volume) and the reader is referred there for further details.

\section{Instrument Response}

\subsection{Multilayer Optics}

The multilayers were deposited on the EIT mirrors at the Institut d'Optique, Centre Universitaire d'Orsay, France. This work is described by Chauvineau et al. (1991). and Delaboudinière et al. (1992). Three of the four quadrants on each of the primary and secondary mirrors were masked to allow multi- 

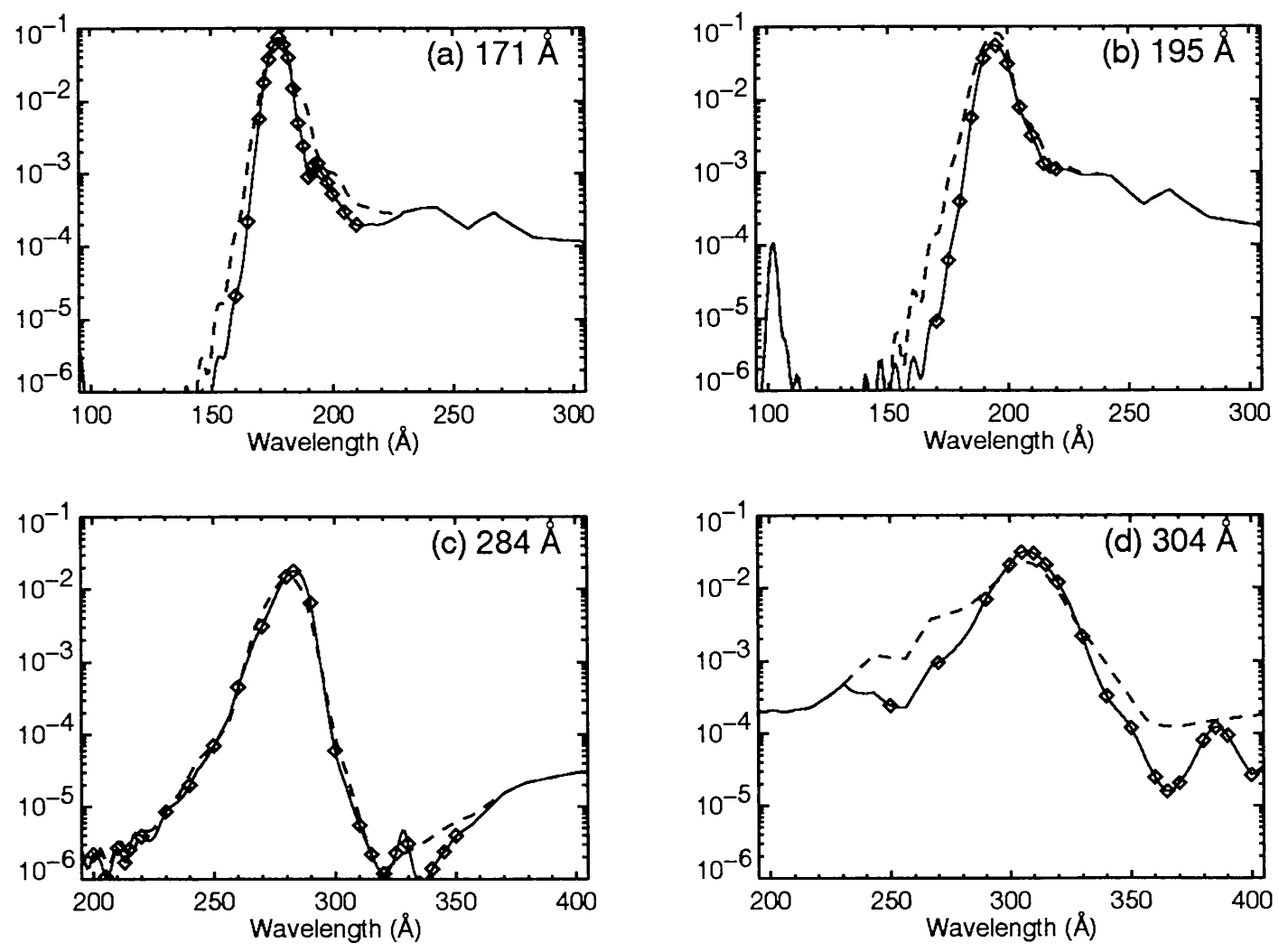

Fig. 5. The two-mirror bounce reflectivity fraction of each EIT quadrant. Measured values are shown as data points; dashed and solid curves represent the predicted and adopted values, respectively.

layer deposition on only the selected quadrants. A witness mirror was placed in close proximity to a flight telescope mirror during each deposition. Thus, the reflectivities of these witness mirrors should closely match those of the telescope mirrors except for a small shift in wavelength of known magnitude. Reflectivities of the witness mirrors were measured at both the BESSY storage ring in Berlin and the facility in Orsay. The product of the primary and secondary witness sample reflectivities for each quadrant are shown by the data points in Figures $5 \mathrm{a}-5 \mathrm{~d}$. The dashed curves in these figures are the response curves calculated from the design parameters of the multilayers and the optical constants of Windt et al. (1988a and 1988b). Except for the case of the $284 \AA$ quadrant, these calculated curves do not fit the witness mirror data well at wavelengths away from the bandpass peak. This is likely due to small departures of layer thicknesses from the design thicknesses during the multilayer deposition. Also, the electron beam deposited materials, in this case, may have slightly different optical constants from those measured by Windt et al. on sputtered materials. The solid curves are the calculated reflectivities, except the resulting curves have been forced to fit the witness sample data. The fully assembled flight telescope reflectivities were also measured and are presently being analyzed. These data will be 


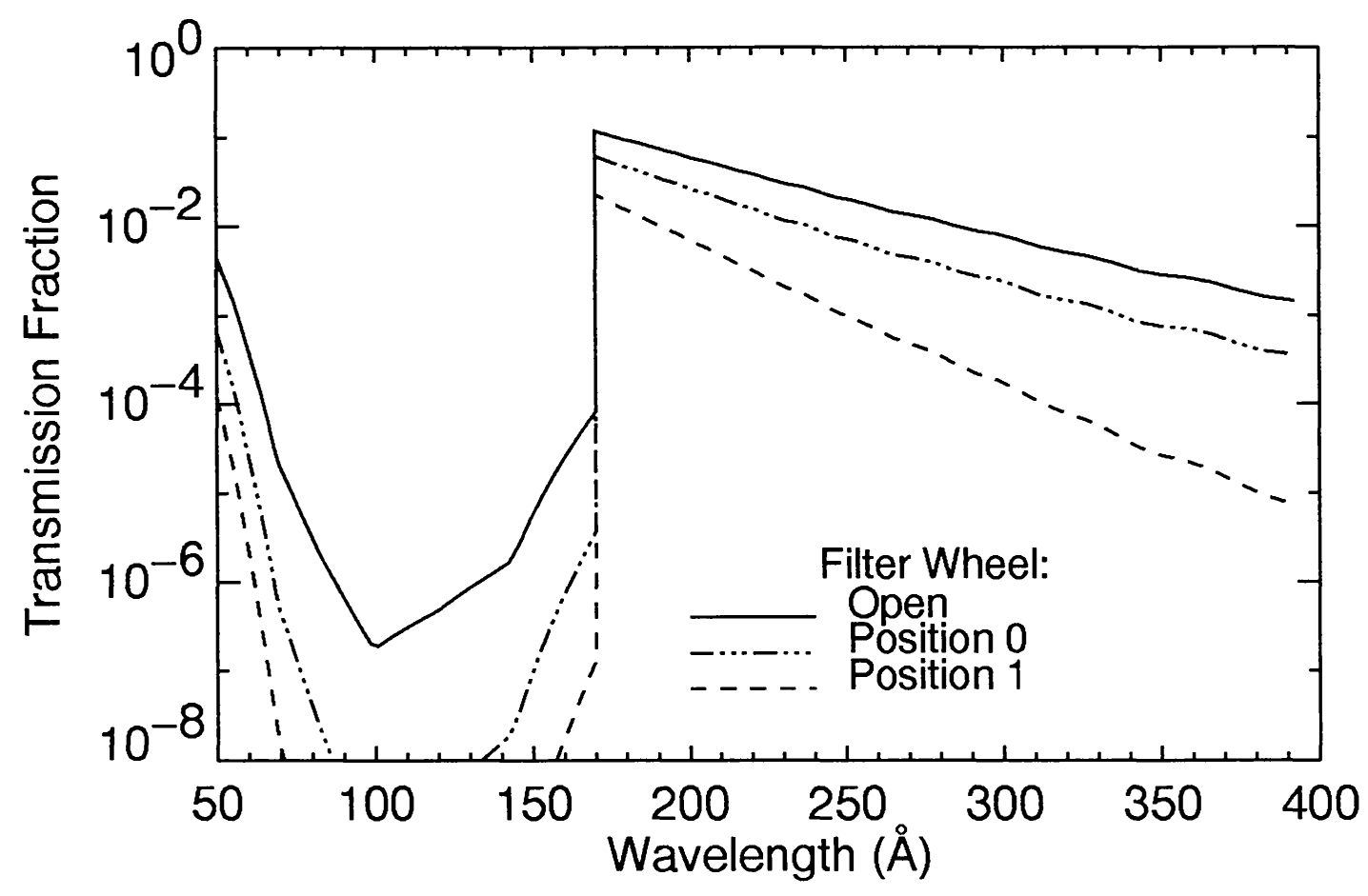

Fig. 6. Calculated transmissions of the EIT thin-film filters.

used to produce the multilayer response curves for analysis of the EIT solar images.

\subsection{Filter Transmissions}

Each quadrant of the primary is protected by a filter which includes two independent sections. Each of the sections has a slightly different transmission due to variance in the manufacturing process. These transmissions have been measured in the telescope at the nominal peak of each bandpass. For the sake of simplicity we only report here on average transmissions which are typical, but slightly different than the flight filters.

The transmission of the filters listed in Table II have been calculated by using EUV absorption coefficients inferred from optical constants derived by Henke et al. (1993) and are shown in Figure 6 as a solid curve. In these calculations a $40 \AA$ layer of $\mathrm{Al}_{2} \mathrm{O}_{3}$ was assumed to have formed on the exposed surfaces of the aluminum. Since the entrance filter and the CCD stray light filter are fixed in the optical path, only the product of their transmissions are shown in Figure 6. This curve is the appropriate filter transmission for the filter wheel in the open position. Also included in the figure are the total optical path transmissions for the filter wheel in positions 0 and 1 , listed in Table II. 


\subsection{CCD Quantum Efficiency}

The quantum efficiency (QE) of the flight CCD has been measured at wavelengths in the range from $171 \AA$ to $800 \AA$ as indicated by the data in Table III.

TABLE III

EIT CCD Quantum Efficiency.

\begin{tabular}{cc}
\hline Wavelength $(\AA)$ & QE \\
\hline 171.4 & 0.36 \\
195.0 & 0.34 \\
284.0 & 0.29 \\
303.8 & 0.27 \\
550.0 & 0.20 \\
800.0 & 0.02 \\
\hline
\end{tabular}

The CCD was illuminated by monochromatic beams of the selected wavelengths and in each case the total net charge in the CCD was measured. The total number of incident EUV photons was measured with a silicon diode calibrated by the National Institute of Standards and Technology. The average number of electron-hole pairs that a photo-electrically absorbed photon of energy $E$ produces in silicon is given by $E / 3.65 \mathrm{eV}$. Thus, from the number of photons incident on the CCD, one may calculate the charge expected to be generated in the CCD if it were 100 percent efficient. The QEs shown in Table III are the ratio of the measured charge to this expected charge. It is always less than unity due to effects that occur at or near the surface of the back-illuminated CCD. Some of the EUV photons are lost by absorption in a thin dead layer on the CCD surface. The electric field in the CCD that sweeps the charge toward the gates on the front surface is weak or possibly reversed near the back surface and some charge may be lost to recombination in this region.

A model of CCD QE, developed by Stern et al. (1994) for a different EUV-enhanced backside-illuminated CCD, was fitted to the data points. They were reasonably well fitted by this model in the range from $171 \AA$ to $550 \AA$. However, the derived CCD parameters were unreasonable, probably because of unknown material components in the proprietary SITe EUVenhancement process. More work must be done to be confident of the QE inferred by the model outside this wavelength range.

Before meaningful analysis of EIT images can be performed, the spatial non-uniformities in the CCD response must be calibrated. The necessary data to provide this flat-field calibration were obtained by illuminating the 


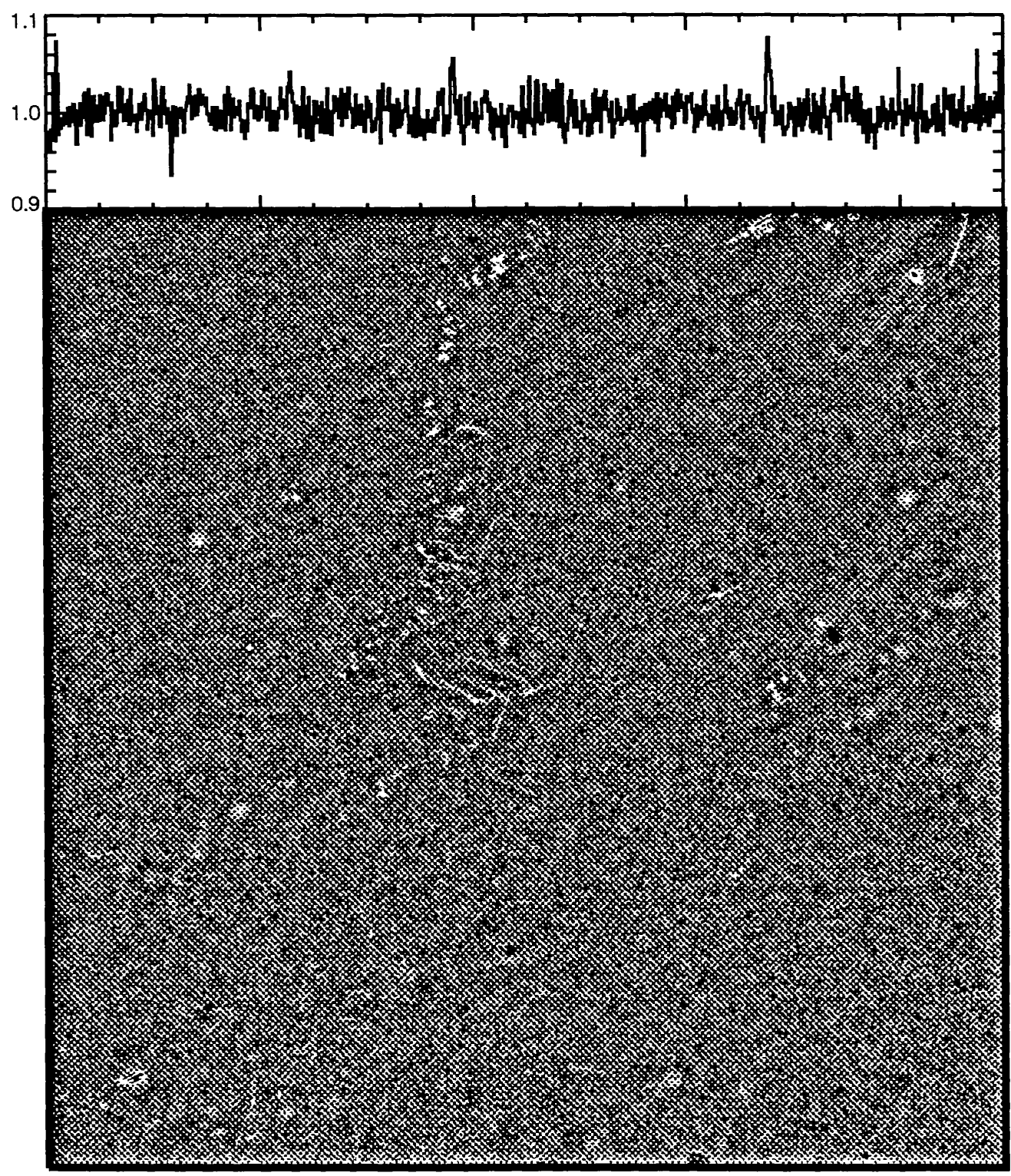

Fig. 7. Flat-field calibration image of the flight EIT CCD. The line trace shows the response for the middle row of the CCD.

CCD with a beam of monochromatic EUV radiation for each of the central wavelengths of the EIT bandpasses. Several images at each wavelength were obtained with the CCD at different positions in the beam. These data may then be used to separate spatial non-uniformities in the beam from variations in CCD response. An algorithm developed by Kuhn, Lin and Loranz (1991) has been used for analyzing these data to obtain the flat-field calibrations. Their formalism works even for substantial spatial non-uniformities in the EUV beam. The flat-field calibration image for the response to $171 \AA$ photons is shown in Figure 7. Raw images obtained in the $171 \AA$ bandpass will be divided by this image to remove spatial non-uniformities. This image is nearly uniform, which illustrates the good flat-field characteristic of the flight EIT device. The line trace shows the response of the row running from 

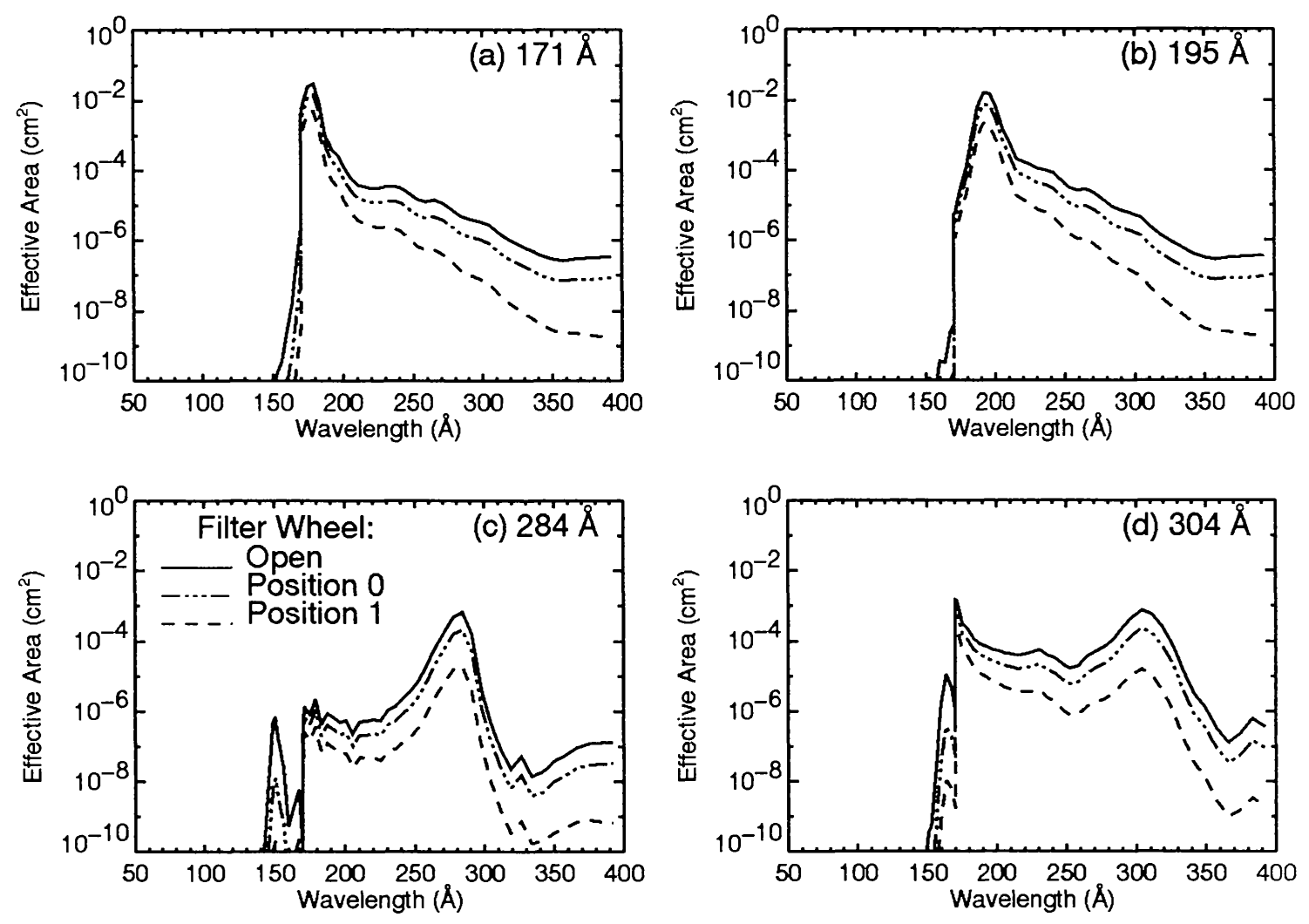

Fig. 8. Effective area of the EIT for each of the four quadrants.

left to right through the middle of the CCD. The calibration image is normalized to unity and has a minimum and maximum of 0.5 and 1.3, respectively, however, $99 \%$ of the pixels have responses that are within $1.0 \pm 0.055$ and $99.9 \%$ of the pixels have responses that are within $1.0 \pm 0.116$. The frequency distribution of the flat-field calibration image is nearly Gaussian: the histogram of the distribution has a full width at half maximum of $3 \%$ and the standard deviation of the entire calibration image is $1.6 \%$.

\subsection{EIT Effective Areas}

The effective areas of each telescope quadrant are shown in Figures 8a-8d. These are the product of mirror reflectivities, filter transmissions and the $13 \mathrm{~cm}^{2}$ geometrical area of each quadrant. The plots show the three filter combinations of Figure 6 and include the CCD efficiency. Note that in Figure 8 the plots extend over larger wavelength ranges than those in Figure 5. This is necessary because some solar radiation, quite distant from the bandpasses, may contribute appreciably to the EIT images. The reflectivities outside the ranges of Figure 5 have been calculated from design parameters of the multilayers and smoothly merged to fit the data points at the extremes of the wavelengths in those plots. More data are available from the flight telescope 
and will be used to calibrate the extended wavelength range for analysis of EIT flight images.

\section{Instrument Control Options}

The EIT has a number of instrument control options that may be selected by commands uplinked from the ground via SOHO telemetry. These include bandpass selection, exposure length, filter selection, CCD readout control and on-board data processing options. Table IV gives a summary of these options.

Sub-arrays of pixels, each $32 \times 32(83 \times 83$ arcsec $)$ in size, may be read out while the rest of the CCD field is discarded. This allows the limited EIT telemetry to be used for detailed study of small areas of the Sun with higher time resolution. This option is implemented by clocking the parallel transfer gates without driving the serial gates until the first row of the region of interest is ready to enter the serial registers. Charge clocked into the serial registers during this period must be cleared by clocking the serial gates 10 times prior to reading the pixels of interest. After the first line of the selected sub-array enters the serial registers the readout proceeds normally until the last line is read. The rest of the image is then discarded as before. Data from pixels in the same lines as the sub-array but lying outside the columns of interest are discarded in the on-board processor. The time required to advance charge in the parallel registers by one line is $480 \mu \mathrm{sec}$. The time required to read out a line of pixels is $21 \mathrm{msec}$. Thus, the time required to read a sub-array contained in $n$ lines of the CCD is $0.0208 n+0.492 \mathrm{sec}$, where $0.492 \mathrm{sec}$ is the time required to clock the parallel registers for reading out the entire array. Since the minimum value of $n$ is 32 , the minimum time between measurements of the sub-array in this mode is about $1.1 \mathrm{sec}+$ the exposure time $+(m \times 0.492) \mathrm{sec}$, where $(m \times 0.429) \mathrm{sec}$ is the time to clear the CCD. In ground-based testing $m=10$ has been used. It should be noted that the $32 \times 32$ subarrays readout in any given observation need not all be contiguous. This allows monitoring more than one region in the field of view without the overhead of reading out all the CCD lines.

\section{Predicted Response to Solar Phenomena}

EIT is capable of imaging solar plasma over a temperature range of 0.06 to $3 \mathrm{MK}$. The sensitivity of EIT for detecting thermal emission from solar plasmas is shown in Figure 9. The curves were computed by convolving the instrument response functions of Figure 8 with theoretical solar X-ray spectra. The continuum was calculated from Mewe et al. (1986). The line rates were taken from an, as yet, unpublished version of the SPEX95 code (Kaastra and Mewe, private communication) which is an update of Mewe, 
TABLE IV

EIT Instrument Control Options.

\begin{tabular}{|c|c|}
\hline \multicolumn{2}{|c|}{ CCD and Mechanism Control } \\
\hline Bandpass Selection & 1 of 4 by open quadrant shutter \\
\hline $\begin{array}{l}\text { Exposure length } \\
\text { mechanical shutter }\end{array}$ & $\begin{array}{l}\text { minimum } 140 \mathrm{~ms} \text {, arbitrary length in } 31 \mathrm{~ms} \\
\text { increments; typical lowest exposure is } 1.5 \\
\text { sec (the shorter exposures require a special } \\
\text { operational mode) }\end{array}$ \\
\hline CCD frame transfer & $0.25 \mathrm{sec}$ \\
\hline Filter selection & Filter wheel, filters (cf. Table II) \\
\hline CCD readout & Full disk, full resolution \\
\hline On-chip summing & $\begin{array}{l}\text { Full disk, half resolution } \\
\quad(2 \times 2 \text { pixel summed }) \\
\text { Full disk, quarter resolution } \\
\quad(4 \times 4 \text { pixel summed })\end{array}$ \\
\hline Partial frame images & $\begin{array}{l}\text { sub-array size and location selectable in } \\
\text { blocks of } 32 \times 32 \text { pixels }\end{array}$ \\
\hline
\end{tabular}

On-board processing: Data compression

Geometric Techniques

BadColAvg: Replace 'hot' column containing CCD defect with average of adjacent columns to allow maximum compression by further techniques.

InterMin: remove the effect of cosmic ray ionization paths in the CCD by basically the same technique and for the same reasons as BadColAvg.

Mask: arbitrary pattern can be transmitted by specifying a mask table of image blocks not to be transmitted.

Subregion: read out only a specified rectangular region of the CCD. The subregions must be constructed of $32 \times 32$ pixel image blocks (which also requires that the beginning pixel location is an integral multiple of 32 ).

PixSum: sum pixels on readout of CCD, typically no more than $4 \times 4$ pixel sums.

Coding Compression Techniques

Rice algorithm (lossless)

ADCT: adaptive discrete cosine transform (lossy) 

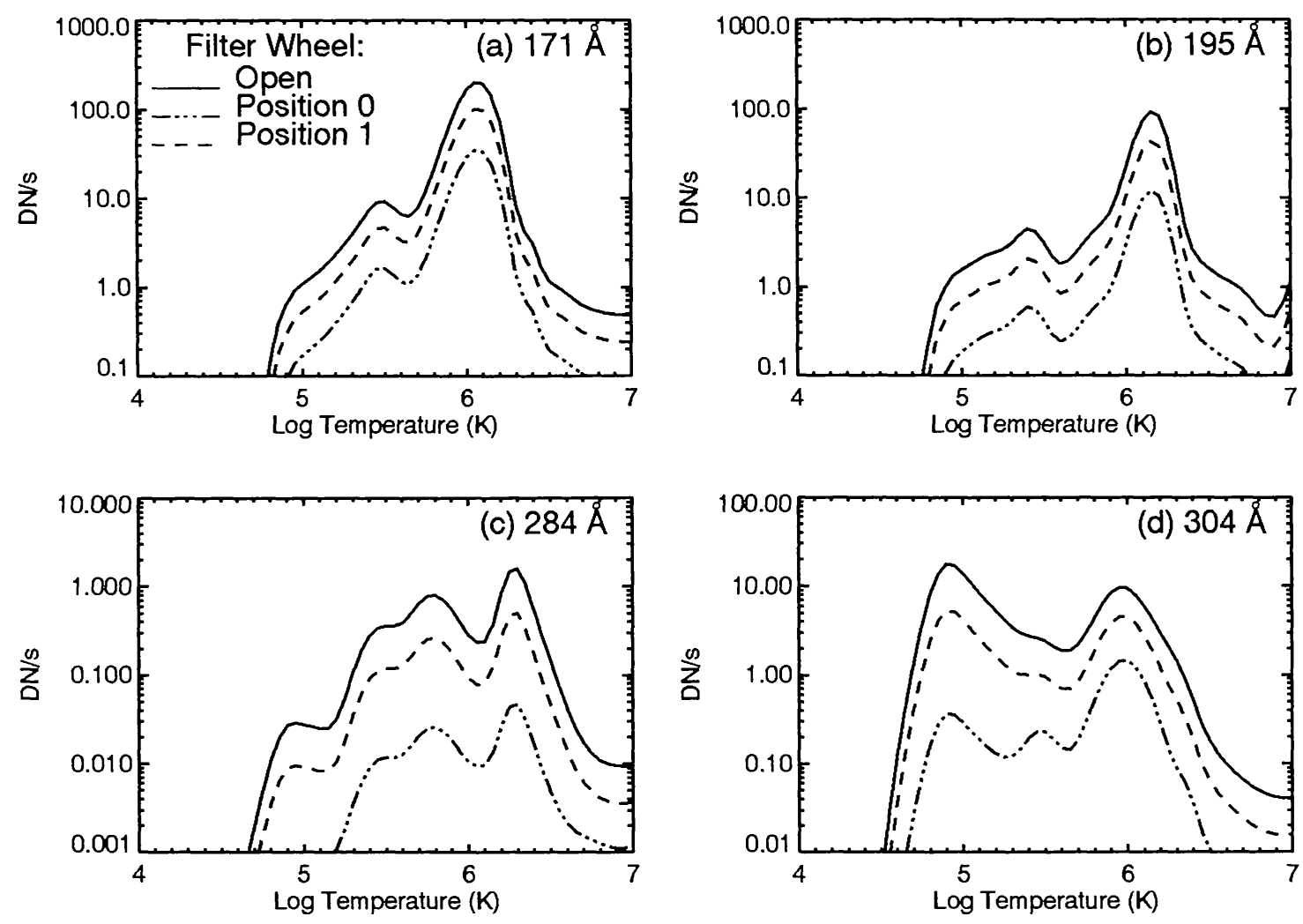

Fig. 9. Signal in the CCD $(D N / s)$ from each of the EIT quadrants as a function of source temperature for a solar emission measure of $10^{44} \mathrm{~cm}^{-3}$.

et al. (1985). For the line emission computations we assumed the ionization balance of Arnaud and Rothenflug (1985) and solar abundances of Meyer (1985). The curves are for an emission measure of $10^{44} \mathrm{~cm}^{-3}$ in units of $\mathrm{DN} / \mathrm{s}$ at the distance of the $L 1$ Lagrangian point; one $\mathrm{DN}$ corresponds to 18 electrons per pixel detected in the CCD.

The panels of Figure 9 show that EIT will have a different temperature response depending on the selected wavelength bandpass. Images obtained from two or more quadrants can be combined to estimate the temperature and emission measure of the coronal material. The solid curves are for the case of the open position in the filter wheel and the dashed curves for filter wheel positions 0 and 1 as in Figures 6 and 8. In Figure $9 \mathrm{~d}$ the response shows a peak for a temperature of $\log \left(T_{e}\right)=4.9$ or $80,000 \mathrm{~K}$ and a second slightly weaker peak at about $1 \mathrm{MK}$. The low temperature peak is due to the He II line at $304 \AA$. The higher temperature peak is caused by Fe IX at $171 \AA$ and the Si XI line at $303.3 \AA$. The EIT response has been estimated in terms of a predicted signal for different types of solar phenomena in Table $\mathrm{V}$. Because solar emission of He II is not necessarily optically thin, the curve in Figure 9d represents an upper limit and for this reason, predictions for the $304 \AA$ bandpass are omitted from Table V. 
TABLE V

Examples of EIT response.

\begin{tabular}{lccccrrr}
\hline Feature & $\begin{array}{c}\text { Temp. } \\
(\mathrm{MK})\end{array}$ & $\begin{array}{c}\text { Em. meas. } \\
\left(\mathrm{cm}^{-3}\right)\end{array}$ & $\alpha$ & $\begin{array}{c}\text { Exp. } \\
(\mathrm{s})\end{array}$ & $\begin{array}{r}171 \AA \\
\text { Signal }(\mathrm{DN}) \text { in one pixel }\end{array}$ \\
\hline Coronal hole & 1.3 & $2 \times 10^{42}$ & 1 & 20. & 70 & 30 & $<1$ \\
X-ray bright point & 1.8 & $7 \times 10^{43}$ & 1 & 10. & 179 & 320 & 10 \\
Coronal loop & 2.1 & $1 \times 10^{43}$ & 10 & 60. & 4 & 8 & $<1$ \\
Active region & 2.5 & $3 \times 10^{45}$ & 144 & 60. & 40 & 41 & 7 \\
Impulsive C flare & 12.0 & $5 \times 10^{48}$ & 10 & 0.5 & 1200 & 5032 & 24 \\
\hline
\end{tabular}

Note: $1 / \alpha$ is the assumed fraction of emission measure detected in one EIT pixel.

\section{EIT Observational Programs and Operations}

\subsection{Observational Programs}

\subsubsection{Full Disk Observations}

EIT will produce a record of the corona over a multi-year period to permit studies of its evolution with the solar cycle and the relationships of its structure and physical properties to the initiation of the solar wind and to events such as coronal mass ejections that produce disturbances in the heliosphere. To obtain this synoptic archive, EIT will observe its full field of $45 \times 45$ arcmin in each of its four wavelength bands at least once per calendar day, and possibly more frequently, depending on the data compression method that is selected. One image per day at each wavelength will be supplied to the SOHO Summary Data File, so that scientists not directly associated with the SOHO mission will have access to overall views of the corona as quickly as is feasible. It is unlikely that the images in the Summary Data File will have the quality of definition and calibration that the fully processed data will have, but they will be adequate to provide an overview of the EUV corona to many non-specialists and the interested public.

The full-disk cadence will be programmed to operate when there is no contact with the SOHO spacecraft. However, full-disk images may also be acquired at the beginning of each long SOHO contact period when EIT and LASCO are allotted an enhanced telemetry rate to support CDS and SUMER science planning activities.

\subsubsection{Selected Areas Mode}

The SOHO telemetry assigned to EIT restricts its solar coverage to, at most, a few full images per day. In order to achieve temporal resolution adequate to record the evolution of specific features such as the emergence and evo- 
lution of coronal loops and processes of energy transport and deposition, the evolution of coronal holes, and the signatures of instabilities leading to coronal mass ejections, EIT plans to execute observations on selected solar areas with a temporal resolution appropriate to the phenomena being investigated. Generally, EIT will observe sequences with a temporal cadence of several minutes between observations, although shorter cadence intervals may at times be possible. The LASCO on-board computer enables the EIT CCD to be read out in blocks of $32 \times 32$ pixels (nominally $83.2 \times 83.2$ arcsec). The locations of such blocks are fixed on the CCD format (there are $32 \times 32$ blocks in the full $1024 \times 1024$ CCD pixel format), But single or multiple blocks may be read out to define a field of view appropriate for the phenomenon being observed.

\subsubsection{Onboard Desaturation}

Despite the large dynamic range of the EIT CCD camera, the dynamic range of the solar corona in the Fe IX, XII, and XV lines is much greater. Since EIT is grossly telemetry bandwidth-limited, it has proven useful to institute an onboard technique to re-expose at a shorter exposure time those $32 \times 32$ pixel subarrays that appear saturated in a preceding exposure. Thanks to the flexibility of the LEB software, this is achieved by the use of an existing masking capability.

\subsection{Coordinated Observing Programs}

EIT will play an important role in supplementing the detailed spectroscopic measurements obtained by CDS and SUMER with images of the coronal features being studied. Coordinated observations will be executed principally during the eight hour contact period, as selection of appropriate coronal features (and near real-time adjustments of the fields of view of the CDS and SUMER instruments) is essential for the success of these studies.

\subsection{EIT OpERATIONS}

\subsubsection{General}

EIT will be operated from the Experimenters' Operations Facility (EOF) located at the Goddard Space Flight Center, Greenbelt, MD. Operations will consist of the preparation, verification and transmittal of commands designed to carry out the desired observational sequences with the instrument. EIT's operational sequences are interleaved with LASCO commands and are, therefore, an integral part of the operational command sequences provided by LASCO to the EOF. As a consequence, timing of all EIT operations, such as operations of motors, interrogation of the CCD, on-board data compression and formatting of the data stream, are integral with LASCO operations and cannot be altered on short notice. An exception is the specification of solar coordinates of subsets of each image recorded on the EIT 
CCD. Such sub-images can be selected by uplink commands to the LASCO command memory during real-time observing programs coordinated with other SOHO instruments such as CDS and SUMER.

\subsubsection{Planning Tools}

EIT, as several other of the instrument teams resident at the EOF, will use an interactive, graphic planning tool to schedule scientific objectives and specific observing programs designed to gather data to meet those objectives. This tool is similar to that described by St. Cyr et al. (1995, this volume), but, as does the LASCO planning tool, includes information on how much data are queued up in the LEB telemetry buffer, waiting to be written to spacecraft onboard memory or downlinked (during realtime contacts). This allows the planner to see whether any observation would overfill the buffer, interfere with the EIT/LASCO synoptic plan, or have to be rescheduled in order to resolve such potential conflicts.

Starting with the synoptic plan, an EIT plan is built by adding additional pre-planned observations (see below), and the resulting plan is passed from the EIT planning workstation to the LASCO workstation. The joint observing plan is iterated until all conflicts are removed, and then uplinked by the LASCO commanding workstation. Note that the EIT ground operations team does not have an independent commanding capability.

\subsubsection{Routine Operations}

Routine operations are those that are executed on a regular basis, usually on a daily basis with a minimum of alterations in the command sequences from day to day. For EIT, the baseline, synoptic observations will be full field of view images in all four EUV bandpasses, taken at least once, and if possible three times per operational day. Such operations will be considered part of the joint EIT/LASCO synoptic plan, and will add no planning overhead on a daily basis.

Observations taken with a higher telemetry rate allocation during a thirty minute interval at the beginning of the 8-hour realtime contact period, for the purpose of providing current EIT and LASCO images to CDS and SUMER, will also be regularly scheduled. Nominally, these images will form part of the synoptic observations just described, but if smaller image areas are desired as well, their size and location must be specified in joint planning sessions with operations personnel from the other instruments (cf. St. Cyr et al. 1995, this volume).

\subsubsection{Other Pre-planned Operations}

EIT will also execute observations of sub-areas of the Sun designed to explore the evolution of coronal features over a wide range of temporal scales. Such operations will be preplanned and executed in conjunction with LASCO 
operations. Image cadence (temporal resolution) is limited by restrictions on computer resources. (EIT is allocated about $20 \%$ of LASCO's on-board resources) and limitations on the total number of operations of EIT mechanical components (shutter, filter wheel, aperture control) over the anticipated lifetime of the mission.

\subsubsection{Realtime or Near-Realtime Operations}

Because of the interleaving of EIT and LASCO operations, a full real-time flexibility in operating EIT is not possible. Operational sequences (wavelength selection, image cadence, etc.) will again be preprogrammed, but the selection of targets (which for EIT means the designation of which sub-areas of the CCD will be transmitted) will be decided in collaboration with other SOHO experimenters. It is expected that sub-area selection may be altered relatively quickly in rapid response to transient activity, other unexpected solar phenomenon, or to optimize joint scientific operations with the other instruments. Such operations will take place only during the extended ( 8 hour) contact period, and the sub-areas to be imaged cannot be larger than those pre-planned.

\subsubsection{Data Compression}

It is currently anticipated that, after initial testing during the spacecraft cruise and commissioning phases, all EIT images will be compressed using the Adaptive Discrete Cosine Transform (ADCT) compression mode. To do otherwise would reduce the amount of data EIT could collect by a factor of 5-10. Typically, the ADCT introduces noise only into the faintest areas of a high-contrast image, and even that noise should be far below the calibration accuracy we can expect to assure for EUV images. In fact, the ADCT algorithm is effective up to compression factors of $16-20$, but throughput limitations in the LEB make it impractical to compress by more than a factor of $10: 1$.

\subsection{Operational Constraints on EIT}

Operational constraints are imposed by the facts that EIT command and data streams are integral with those of LASCO, by the limited telemetry allocated to the EIT experiment and that LEB operations are serial in nature. In general, the guidelines imposed by these constraints are:

- Only one motor (in either LASCO or EIT) may be operated at a time.

- Only one camera (in either LASCO or EIT) may be read out at a time.

- EIT will use, on average, about $20 \%$ of the total telemetry capability and computer resources available to the EIT/LASCO experiments.

- One full-field, full-resolution EIT image (or equivalent) may be stored in the on-board memory without data compression. Data compression routines that are available in the EIT/LASCO on-board computer allow 
the storage/transmission requirements for an image to be reduced by a factor of 2-4 using a loss-less compression such as a square root or a "Rice" algorithm. A compression factor of up to 10 may be obtained by the use of the ADCT algorithm, if some loss of information is acceptable.

\section{Conclusion}

The EIT is a very versatile instrument that will provide full-Sun EUV images covering temperatures ranging from the chromosphere into the corona. EIT's spatial (2.6 arcsec pixels) and diagnostic capabilities will enable it to study a wide range of coronal problems and will enhance the studies made by other diagnostic instruments on the SOHO spacecraft. The EIT images will provide an important link between observations of the inner and outer corona and will also serve to correlate SOHO data with ground-based observations.

\section{Acknowledgements}

The French laboratories (IAS, IOTA and LAS) were supported by CNRS and CNES. The Belgian laboratories were funded by SPPS via PRODEX. NRL received support from the Office of Naval Research and from NASA through contract DPR S-19506-E. LPARL acknowledges NASA support through contract NAS5-32627 and NRL contract N00014-90-C-2346 and from the Lockheed Independent Research Program. We benefited greatly from the work of Dr. Barry Labonte of the Yohkoh SXT team who provided an IDL version of the flat-field algorithm of Kuhn et al. (1991). We thank R. Nakatsuka, E. E. Einfalt and S. L. Freeland for aid in developing the ground data analysis system and software. G. Holland contributed greatly to this project by his extensive innovation in vacuum related technologies. We are indebted to the Scientific Imaging Technologies Inc., Tektronix for fabricating the CCDs used in this investigation. We acknowledge the work of the Physikalisch-Technische Studien G.M.B.H. (Germany) and the Luxel Corporation (USA) in fabricating the thin-film filters for the EIT.

\section{References}

Arnaud, M., Rothenflug, R.: 1985, Astron. Astrophys. Suppl. Ser. 60, 425

Bottema, M., Woodruff, R. A.: 1971, Appl. Opt. 10, 300

Brueckner, G. E., Howard, R. A., Kooman, M. J., Korendyke, C. M., Michels, D. J., Socker, D. G., Dere, K. P., Lamy, P. L., Liebaria, A., Bout, M. V., Schwenn, R., Simnet, G. M., Bedford, D. K., Eyles, C. J.: 1995, Sol. Phys., this volume.

Catura, R. C., Shing, L.: 1993, Proc. SPIE 2006, 286

Chauvineau, J. P., Clotaire, J. Y., Colas, G., Lam, O., Manneville, J. C., Marioge, J. P., Mullot, M., Raynal, A., Tissot, G., Valiergue, L., Delaboudinière, J. P.: 1991, Proc. SPIE 1546, 576

Clette, F., Delaboudinière, J.-P., Dere, K. P., Cugnon, P.: 1995, 'EIT: The Extreme Ultraviolet Imaging Telescope' in Arnold O. Benz and Albrecht Krüger, ed(s)., 
Coronal Magnetic Energy Releases, Proceedings of the CESRA Workshop, Springer: Caputh/Potsdam, Germany, 251

Delaboudinière, J.-P., Gabriel, G. H., Artzner, G. E., Michels, D., Dere, K., Howard, R., Catura, R., Stern, R., Lemen, J., Neupert, W., Cugnon, P., Koeckelenbergh, A., Van Dessel, E. L., Jamar, C., Maucherat, A., Chauvineau, J. P., Marioge, J. P.: 1989a, ESA SP-1104, 43

Delaboudinière, J.-P., Gabriel, G. H., Artzner, G. E., Millier, F., Michels, D., Dere, K., Howard, R., Kreplin, R. W., Catura, R., Stern, R., Lemen, J., Neupert, W. M., Gurman, J. B., Cugnon, P., Koeckelenbergh, A., Van Dessel, E. L., Jamar, C., Maucherat, A., Chauvineau, J. P., Marioge, J. P.: 1989b, Proc. SPIE 1160, 518

Delaboudinière, J.-P., Hochedez, J. F., Chauvineau, J. P., Valiergue, L.: 1992, Proc SPIE 1742, 296

Henke, B. L., Gullikson, E. M., Davis, J. C.: 1993, Atomic Data and Nuclear Data Tables Vol. 54, No. 2

Kuhn, J. R., Lin, H., Loranz, D.: 1991, PASP 103, 1097

Mewe, R., Gronenschild, E. H. B. M., van den Oord, G. H. J.: 1985, Astron. Astrophys. Suppl. Ser. 62, 197

Mewe, R., Lemen, J. R., van den Oord, G. H. J.: 1986, Astron. Astrophys. Suppl. Ser. 65, 511

Meyer, J.-P.: 1985, Ap. J. Suppl. Ser. 57, 173

Moses, D., Howard, R., Wang, D., Catura, R. C., Lemen, J., Shing, L., Stern, R. A., Hochedez, J.-F., Delaboudinière, J.-P.: 1993, SPIE Proc. 2006, 252

Spiller, E.: 1994, Soft X-Ray Optics, SPIE Optical Eng. Press, Bellingham, WA

St. Cyr, O. C., Sánchez-Duarte, L., Martens, P. C. H., Gurman, J. B., Larduinat, E.: 1995, Solar Phys., this volume.

Stern, R. A., Shing, L., Blouke, M. M.: 1994, Appl. Opt. 33, 2521

Tsuneta, S., Acton, L., Bruner, M., Lemen, J., Brown, W., Caravalho, R., Catura, R., Freeland, S., Jurcevich, B., Morrison, M., Ogawara, Y., Hirayama, T., Owens, J.: 1991, Solar Phys. 136, 37

Windt, D. L., Cash, W. C. Jr., Scott, M., Arendt, P., Newnam, B., Fisher, R. F., Swartzlander, A. B.: 1988a, Appl. Opt. 27, 246

Windt, D. L., Cash, W. C. Jr., Scott, M., Arendt, P., Newnam, B., Fisher, R. F., Swartzlander, A. B.: 1988b, Appl. Opt. 27, 279 